\title{
RELATION OF CAMERA ERROR TO PHOTOGRAMMETRIC MAPPING
}

\author{
By Irvine C. Gardner
}

\section{ABSTRACT}

A portion of the errors in a map constructed from airplane photographs arises from differences between the actual performance of the airplane camera and the postulated performance as defined by the calibration constants. The different sources of errors of this nature are enumerated and equations are derived showing the relation between errors in the calibration constants of the camera and the resulting errors in the map.

Equations 3, 4, 10, 11, 18, and 19 give resulting errors in the map arising from incorrect value of the calibrated focal length, distortion, and incorrect location of the principal point. Other sources of error in the camera, such as failure of the film to lie in a plane, the character of performance of the shutter, and imperfect filters are considered and their effects upon the image evaluated.

\section{CONTENTS}

I. Introduction

1. General characteristics of the airplane camera_............ 210

2. Differences between ideal imagery and actual camera performance

3. Sources of error in the map.................... 213

4. General consideration of errors of the camera $\ldots \ldots \ldots$

II. Calibrated focal length _...

1. Permissible range in selection of calibrated focal length $\ldots \ldots \ldots 215$

2. Map errors resulting from error in determining calibrated focal length . . .

3. Causes of variation in calibrated focal length ................ 218

III. Distortion _.

1. Variation of distortion with selection of focal length

(a) Criterion for selecting the calibrated focal length...... 220

2. Error arising from distortion . . . . . . . . . . . . . . 221

3 . Values of focal length and distortion reported for a lens not mounted in a camera

IV. Principal point of the focal plane

1. Index marks for principal point _...

2. Relation between the principal point and the axis of the lens ... 225

3. Error arising from incorrect location of the principal point_..... 226

(a) Case in which the two overlapping photographs are

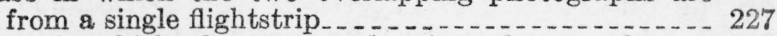

(b) Case in which the two overlapping photographs are from adjacent flightstrips........................ 229

(c) Equations generalized to apply to the two preceding

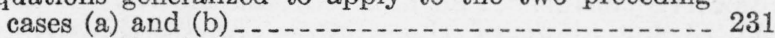

V. Departure of film from the focal plane 
VI. Types of shutters

1. Between-the-lens shutter..... 232

2. Focal-plane shutter........ 233

(a) Efficiency

(b) Distortion introduced by focal-plane shutter....... 234

VII. Accessories in optical path of camera _.

1. Filter

(a) Types of filters

(b) Distortion introduced by filter ................. 237

(c) Effect of filter upon definition and camera calibration. 237

2. Pressure plate _. .

\section{INTRODUCTION}

\section{GENERAL CHARACTERISTICS OF THE AIRPLANE CAMERA}

The airplane mapping camera is a precision measuring instrument. Although fundamentally an optical instrument, it is dependent, for proper functioning, not only upon the proper design and installation of the optical parts but also upon the proper performance of a large number of elaborate and complicated mechanical auxiliary parts, among which are the mount which prevents the vibration of the airplane from affecting the definition of the photograph, the film mechanism by which the film is advanced and constrained to lie in the focal plane, the photographic shutter by which the exposure is timed, and the control devices by which the desired overlap is secured between the different negatives of a series. In this discussion consideration will be restricted to the fundamental requirements that are necessary in order that the resulting negatives may have the metrical characteristics suitable for the production of a map of the desired accuracy. These requirements relate to the precision with which the equivalent focal length must be determined, the distortion introduced by the objective, the location of the principal point of the focal plane, the planeness and shrinkage of the film, the type of shutter, and choice of focal length. After the tolerances connected with these characteristics have been derived and a suitable photographic objective has been chosen, it then becomes the problem of the camera designer to design the mechanical parts of the camera so that the photographic operations may be satisfactorily performed in a manner consistent with the maintenance of these tolerances. The requirements for the mechanical parts, except as they affect optical performance, will not be considered in this discussion.

When use is made of an airplane photograph for the construction of a map, it is commonly assumed that the photograph is a true perspective representation. Usually this is not rigorously true as distortion and other defects prevent the realization of a perfect perspective. Points $A, B, O, C, D$, and $H$ in figure 1 are object points that are imaged on the photograph at $A^{\prime}, B^{\prime}, O^{\prime}, C^{\prime}, D^{\prime}$, and $H^{\prime}$, respectively. The point $N^{\prime}$ that brings the points of the photograph as nearly as possible into the correct perspective relationship should be chosen as the perspective center. The point $O^{\prime}$, at the foot of the perpendicular from $N^{\prime}$ to the plane of the photograph, is the principal point ${ }^{1}$ and the length $N^{\prime} O^{\prime}$ is the principal distance. These terms are standard ones long used in the theory of perspective and are applicable to any representation in perspective, whether it is a photograph, painting, or

1 This principal point of the photograph or of the focal plane is not to be confused with the principal points of the lens, a pair of points coincident with the nodsl points in lenses of the types employed for photogram. metric purposes. 
drawing. It will be noted that the definitions are in terms of the photograph and bear no reference to the camera.

The point $N$ is the corresponding perspective center for the terrain that is photographed. If $\beta$ is the angle at $N$, subtended by $O$ and any point $A$, and if $y^{\prime}$ is the distance from $O^{\prime}$ to $A^{\prime}$, then since, ideally, $\beta=\beta^{\prime}$, it is evident that

$$
\tan \beta=\frac{y^{\prime}}{p},
$$

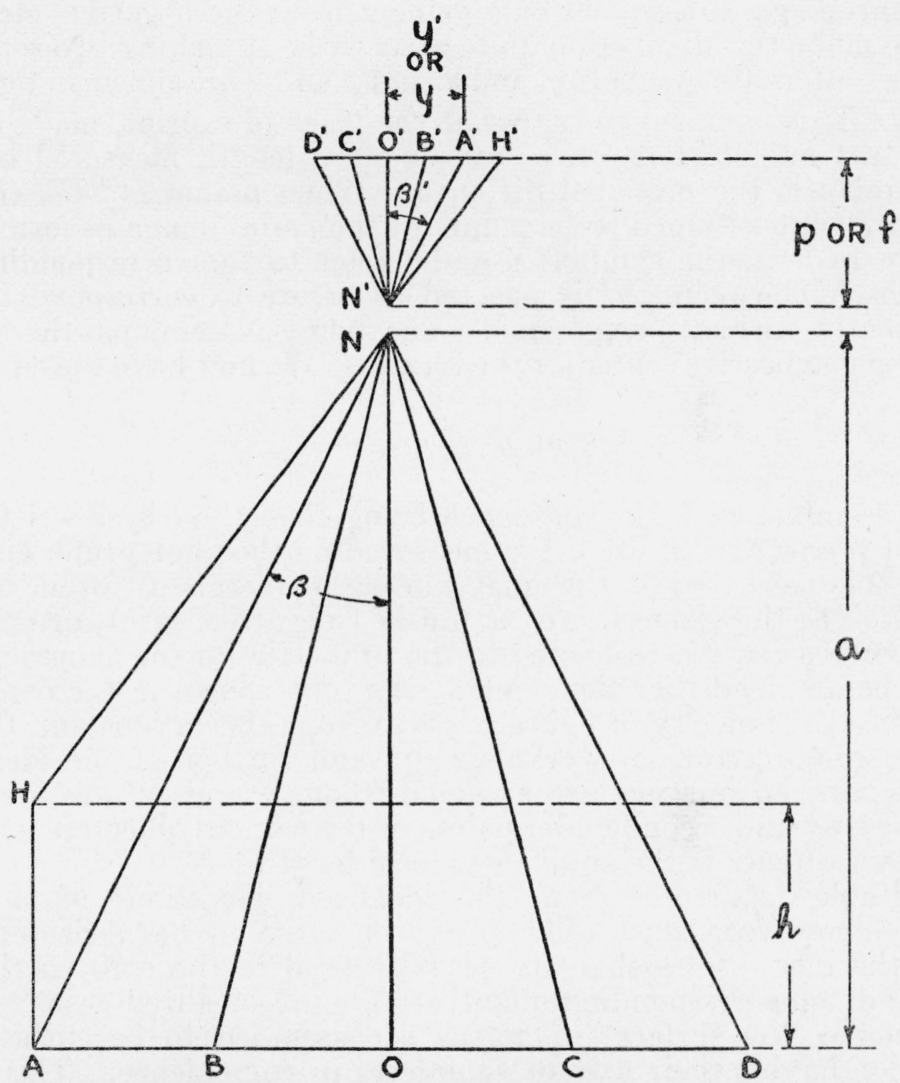

FIGURE 1.-The metrical relations of the camera.

The symbols $y^{\prime}$ and $p$ designate the indicated lengths when $D^{\prime}, C^{\prime}, O^{\prime}, B^{\prime}, A^{\prime}$, and $H^{\prime}$ are assumed to be points on a print or diapositive. If they are assumed to be images of points, as formed in the camera (in which case they are unaffected by shrinkage of film or subsequent enlargement), the symbols $y$ and $f$ apply.

where $p$ is the principal distance. This relationship enables the photograph to be utilized for the measurement of angles. In practice these angles may be determined either by computation, after a direct measurement of $y^{\prime}$, or, if a plotting machine is employed, the angle $\beta$ is determined and utilized to transfer the desired result to the map in a semiautomatic manner without an intermediate reading or recording of the angle. 


\section{DIFFERENCES BETWEEN IDEAL IMAGERY AND ACTUAL} CAMERA PERFORMANCE

Returning to figure 1 , let it now be assumed that points $A^{\prime}, B^{\prime}, O^{\prime}$, $C^{\prime}, D^{\prime}$, and $H^{\prime}$ are no longer the points recorded on a photograph, as was the case in the discussion of the preceding paragraph, but that they are the image points as actually produced in the focal plane by the photographic objective. The distinction lies in the elimination of possible changes in the spacing of the points as a result of film and paper shrinkage, subsequent enlargement from the negative, etc. In order to make this distinction quite clear without making two separate drawings, alternate symbols, $y^{\prime}$ and $y$, and $p$ and $f$, are shown in figure 1 . When $\overline{D^{\prime} H^{\prime}}$ is assumed to represent the trace of a print, made either by contact or enlargement, $y^{\prime}$ represents a length measured on the print and $p$ is the principal distance. When points $D^{\prime}, C^{\prime}, O^{\prime}, B^{\prime}$, $A^{\prime}$, and $H^{\prime}$ are assumed to be points of the aerial image as formed by the camera lens, the symbols $y$ and $f$ refer to the corresponding dimensions. The point $N^{\prime}$ is selected as before to correspond to the most nearly correct perspective. The point $N$ remains the corresponding perspective center for the terrain. We now have the equation

$$
\tan \beta=\frac{y}{f},
$$

which resembles eq 1 , the difference being that $f$ has been written instead of $p$ and that $y^{\prime}$ in eq 1 is measured on the photograph and the $y$ of eq 2 is measured on the image in the camera. In other words, eq 1 involves the properties of a finished negative, print, or enlargement, whereas eq 2 is restricted to the properties of the camera.

If it be assumed that the camera lens (not shown in the diagram) producing the imagery of figure 1 is free from aberrations and the inevitable minor errors of workmanship, and further, if the idealized rays of paraxial imagery are assumed, then $N$ and $N^{\prime}$ are identical with the first and second nodal points or the camera objective and the quantity $f$ of eq 2 is the equivalent focal length. ${ }^{2}$

Inevitable departures from the idealized conditions of paraxial imagery, however, cause differences, too large to be neglected, between the magnitudes that are actually used in the construction of maps and the corresponding quantities for the idealized case.

Ordinarily the surfaces of a lens are assumed to be surfaces of revolution having their axes of symmetry in coincidence. This common axis is referred to as the axis of the lens. When a lens is carefully examined on the test bench, it is often found, even though the lens is of excellent quality and repute, that the axes of the different surfaces fail to coincide exactly. In such a case it is difficult to define the axis of the lens or the positions of the nodal points with precision. If a line approximately coincident with the axes of the several surfaces is tentatively accepted as the axis of the lens, it may be found that a ray in the object space traveling along this line is deviated several min-

2 This is based on the assumption that $a$, the altitude of the camera, may be considered infinite. When $a$ is 15,000 feet and the focal length of the camera objective is 6 inches $(150 \mathrm{~mm})$, the displacement of the image from the rear focal plane, because of the finite, though large distance to the object, is approximately two tenthousandths of an inch, an amount negligible in comparison with the other departures from the ideal condition that are to be considered. 
utes by transmission through the lens. Consequently, $N$ and $N^{\prime}$ of figure 1 have been referred to, not as nodal points of the lens but as centers of perspective in the object and image space. The principal point of the focal plane is not necessarily the point where the axis of the lens intersects the image plane but is at the foot of the perpendicular dropped from the center of perspective in the image space to the image plane. For the pair of conjugate points, $N$ and $N^{\prime}$, angles $\beta$ and $\beta^{\prime}$ are equal when distortion is absent. These points may, therefore, be termed the points of unit angular magnification, although they satisfy this condition rigorously only in the ideal case. As a consequence of this unit angular magnification, a lens may be tipped about $N^{\prime}$ through an appreciable angle without seriously affecting the metrical characteristics of the negative and, in such a case, the point $O^{\prime}$ will not coincide with the intersection of the axis of the lens and the focal plane.

The equivalent focal length is usually defined in textbooks on optics in terms of paraxial imagery, and it is treated as though it were a uniquely defined quantity associated with the lens. Actually, when the physical nature of light and other factors, such as the graininess of the photographic emulsion, are taken into consideration there is a considerable range of positions in which the image plane may be established without marked variation in definition. For each of these settings there is a different value of the $f$ of eq 2 , and this is but one reason why the $f$ of eq 2 is not necessarily identical with the computed focal length. When distortion is present, eq 2 is not valid for all parts of the field unless it is modified by the addition of a correction term. If the plotting machine has no provision for the introduction of such a correction term, a partial correction can be secured by choosing a modified value of $f$ in a manner that is more fully considered in a later section. In view of differences of this nature between the $f$ of eq 2 and the equivalent focal length, the former will be referred to as the calibrated focal length, and it is defined as the value that best satisfies eq 2 for all points of the image.

According to this nomenclature, therefore, the equivalent focal length is an optical characteristic of a lens defined in terms of paraxial imagery; while the calibrated focal length is a property of the lens and camera, which not only depends upon the equivalent focal length of the lens and the amount of distortion present but is also governed by the manner in which the lens is adjusted in the camera. The principal distance is a property of the finished negative or print and differs from the calibrated focal length in that it is further affected by film shrinkage, by the shrinkage of the paper base, if a print has been made, and by any enlargement or reduction. If, for example, a photograph is enlarged any number of diameters, the principal distance is proportionately increased.

\section{SOURCES OF ERROR IN THE MAP}

Errors in the map may result from errors introduced into the photograph by the camera, from changes such as shrinkage of the film or paper, and from errors introduced during the process of making the map from the photograph. This last classification includes faulty enlargement; imperfect rectification or tilt elimination by projection; systematic errors introduced by the plotting machine as a consequence 
of inaccurate gears, pantograph linkages, and projectors; errors systematic for each print but changing from print to print because of incorrect adjustment of photographs in the plotting machine; errors arising from lost motion in the plotting machine; and accidental errors arising from the inability of the operator to make pointings unaffected by accidental variations. Errors of a systematic nature may also arise because of errors in the system of control points.

This presentation is restricted to a consideration of the errors that have their origin in the camera. They are: a, a difference between the assumed and actual value of the calibrated focal length; $b$, distortion produced by the optical system; c, a difference between the assumed and actual position of the principal point; $d$, a departure of the film from a plane; e, distortion introduced by the shutter; and $f$, distortion introduced by filter or pressure plate.

\section{GENERAL CONSIDERATION OF ERRORS OF THE CAMERA}

In evaluating the effects of these sources of error upon the map it is necessary to consider the manner in which the photographs are used. It might at first appear necessary to consider only the displacement or apparent displacement of an image point as produced by those variations and then to determine the corresponding displacement upon the map. This procedure might be followed if the photographs could be used for map making without known control points. In airplane mapping, however, control points are necessary ${ }^{3}$ and consequently the introduction of residual errors in the final map is too subtle to be evaluated so easily by such a simple direct computation which would give estimates larger than the errors actually introduced.

In the interpretation of aerial photographs one makes direct use of the known control points in the picture. If (1) an automatic-plotting machine is used to interpret the negative, one adjusts the negative to fit the control points. In this operation it is usually assumed that there is no distortion and that the camera is correctly calibrated and collimated. Implicitly, this adjustment of the negative in the machine amounts to a determination of the position of the camera in space at the instant of exposure. If (2) the negative is to be interpreted by direct measurements and mathematical analysis, one first determines explicitly the position and tilt of the camera at the instant of exposure. In case (1) or case (2) the choice is made in such a manner"that eq 1 , with the assumed calibration, is valid, or made as nearly valid as possible for all control points. If these assumptions are incorrect an incorrect position for the camera at the time of exposure will be determined but, as has been noted, the error will be such that eq 1 , with the incorrect values that have been assumed, applies to the control points. Consequently, this error in the location of the camera is of a compensatory nature and tends to correct the errors for other points on the negative.4

\footnotetext{
3. One may be actually fitting a photograph to control points or, when "bridging" from one set of control points to another, there may be no control points on a given print, but it may be adjusted to have the proper relation to the preceding photograph of the series. For the purposes of this discussion, however, these two operations are essentially similar in character, whether the print is fitted to control points independently surveyed or to the points of the preceding print.

i This compensating effect occurs in all applications of airplane photography to mapping, and it considerably increases the allowable variations in the performance of the camera. It is interesting to note, that on the contrary when mapping is done by cameras on tripods at the ends of measured base lines the positions of the cameras are observed by direct surveying methods rather than by fitting the negatives to control points and therefore this compensation is not obtained.
} 
The same idea may be expressed, from another viewpoint, by stating that the camera serves as a means for determining the positions of points in space by interpolating or extrapolating from the known control points. If control points lie at the extreme positions of the photographs and at maximum and minimum elevations, then all points are "bracketed" by the control points and the process is entirely interpolation. If this is not the case, some of the points to be located lie beyond the control points and are located by extrapolation. The exact functional relationship by which the interpolation or extrapolation is to be done is known and the only errors incidental to the process are the accidental errors arising from lack of precision in the different steps of the operation and the systematic errors (systematic in the results obtained from a given pair of photographs) arising from the use of incorrect constants, such as calibrated focal length and incorrectly located principal point. Consequently, the results obtained by extrapolation, although probably less accurate than those obtained by interpolation, are not so markedly inferior as may be the case when extrapolation is done by an entirely empirical method.

The distribution of the control points is governed by the nature of the region to be mapped and the results available from preceding surveys. Consequently, the distribution necessarily varies from photograph to photograph, with consequent changes in the errors resulting from departures of the camera from its calibrated values. In this discussion of errors the assumption is made that all the control points lie in a datum plane. The systematic errors arising from errors in the camera are largely independent of the distribution of the control points, but for points located from a given pair of photographs the systematic errors resulting from the necessarily imperfect fitting of the photographs to the control points increase rapidly if the control points are not distributed over the entire region included in the photographs. If all control points lie in a plane, the determination of all elevations is a result of extrapolation, and consequently the errors in stereoscopic parallax resulting from errors in the camera may be expected to be maximal. If the control points are distributed not only in the horizontal datum plane but also over the range of elevation presented by the terrain, it is probable that the errors of stereoscopic parallax will be less than those embodied in the conclusions of this presentation.

\section{CALIBRATED FOCAL LENGTH}

\section{PERMISSIBLE RANGE IN SELECTION OF CALIBRATED FOCAL LENGTH}

It has already been suggested that the value of $f$ in eq 2 that makes this equation most nearly valid for all parts of the negative be designated as the "calibrated focal length" and that this can be expected to differ from the equivalent focal length. For lenses that are not assembled in cameras when submitted to the National Bureau of Standards for test, the reports give values of the equivalent focal length and of the back focal length. If distortion were absent and if, light consisted of rays that could be represented by geometric lines, these values would each be unique. Actually, however, there is considerable depth of focus, and the values given in a report are self- 
consistent values lying near the middle of a range of two or more tenths of a millimeter, the total variation without marked change in definition depending upon the lens and the relative aperture. If a lens that has been tested is mounted in a camera, and if a depth gage is used to make the distance from the vertex of the last surface of the lens to the plane of the emulsion, equal to the reported back focal length, then, in the absence of distortion or tipping of the lens, the calibrated focal length should be equal to the equivalent focal length given in the report. If, however, the distance of the lens from the focal plane is selected by trial exposures made as this distance is varied, it will be fortuitous if the adopted distance from lens to film is the same as the back focal length given in the report. Consequently, even in the absence of distortion, the calibrated focal length of a lens mounted in a camera may not be the same as the equivalent focal length as measured on the same lens submitted unmounted.

A portion of this depth of focus, which results in an image equally good along a considerable range of the optic axis, arises because of the diffraction resulting from the wave character of light. This contribution to the depth of focus varies as the square of the " $f$ number" and, for an ideal lens, has the values given in table 1.

TABLE 1.-Depth of focus as a funciion of aperture

\begin{tabular}{|c|c|}
\hline $\begin{array}{c}\text { Relative aper- } \\
\text { ture }\end{array}$ & Depth of focus \\
\hline & $m m$ \\
$f / 2$ & \pm 0.004 \\
$f / 2.8$ & .008 \\
$f / 4$ & .016 \\
$f / 5.6$ & .032 \\
$f / 8$ & .064 \\
$f / 11$ & .128 \\
$f / 16$ & .256 \\
$f / 22$ & .512 \\
\hline
\end{tabular}

These limits are considerably extended in actual practice because of the graininess of the photographic emulsion, which limits the resolving power of the film. For testing purposes at the National Bureau of Standards, a special spectrographic plate with an emulsion designated by the Eastman Kodak Co. as type $V$ is used. This emulsion has a very fine grain with high resolving power but is too slow for use in the field. Even with this emulsion, exposures made with a relative aperture of $f / 8$ and spaced $0.3 \mathrm{~mm}$ along the axis show scarcely distinguishable differences in sharpness between consecutive positions. This corresponds to a possible variation of $\pm 0.15 \mathrm{~mm}$ in the value of $f$, depending upon the adopted setting of the lens in the camera.

The presence of this depth of focus is often very advantageous. When a lens has considerable curvature of field, it is possible to locate the focal plane in a compromise position for which the definition is greatly improved at the edge of the photograph without materially reducing the quality of definition at the center.

\section{MAP ERRORS RESULTING FROM ERROR IN DETERMINING CALIBRATED FOCAL LENGTH}

To determine how precisely the calibrated focal length need be determined and the magnitude of allowable variations arising from 
temperature change of camera, from removal and replacement of magazine, etc., it is necessary to consider the effect resulting from using an erroneous value of $f$ in constructing a map. A little consideration leads to the conclusion, somewhat startling at first, that so long as the camera is vertical and the terrain a horizontal plane, an error in the value of $f$ introduces no error at all in the map. All such pictures, taken with lenses of different focal lengths or at different heights, are rigorously geometrically similar, and consequently the negatives differ only in scale. When the negative is adjusted in the plotting machine to fit the control points the scale is adjusted to the correct value and the representation is correct.

However, if the terrain is not plane, the relief parallax ${ }^{5}$ will be different for photographs taken from different heights because, as the elevation of the camera is changed, the displacement on the negative, for the points nearer the camera, that is, for the more elevated points, changes disproportionately to that for the less elevated points.

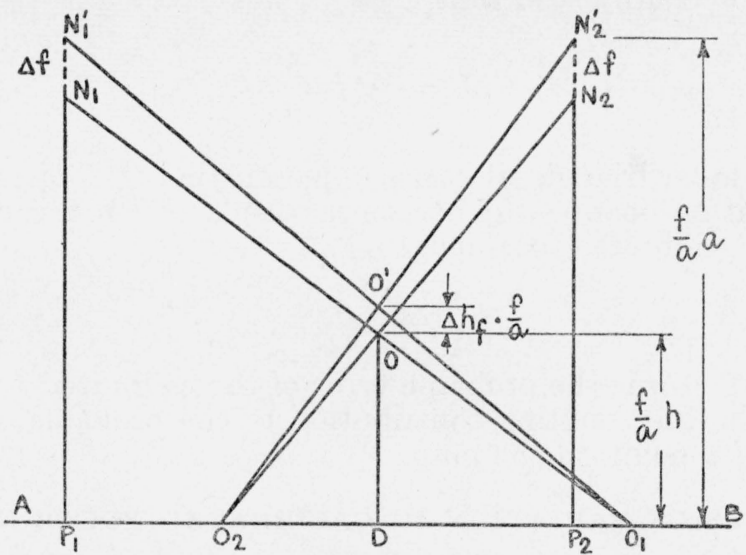

FIGURE 2.-The error arising from an incorrect value of the calibrated focal length.

The correct centers of perspective are at $N_{1}$ and $N_{3}$. Becsuse of the error $\Delta f$ in the calibrated focal length, points $N_{1}^{\prime}$ and $N_{2}^{\prime}$ are assumed to be the centers of perspective and the elevated point $O$ is incorrectly located at $O^{\prime}$, a point displaced vertically but not horizontally.

The effect of an incorrect value of the calibrated focal length is illustrated in figure 2, which is an elevation, $\overline{A B}$ being the trace of two overlapping photographs, 1 and 2 , assumed to be so placed that the common control points coincide. ${ }^{6}$ The principal points are at $P_{1}$ and

6 The relief parallax is the apparent relative lateral displacement of a point because of its elevation or depression from an assumed reference plane. In figure 1 , the point $H$, is directly over $A$, but, in the focal plane, $H^{\prime}$ is laterally displaced with respect to $A^{\prime}$. The displacement, $\overline{H^{\prime} A^{\prime}}$, is the relief parallax and always extends in a radial direction from the principal point. When two overlapping photographs are properly arranged for stereoscopic viewing, the relief parallax gives rise to the stereoscopic relief by which the elevation of a point common to the two photographs can be determined.

6 In this figure and the similar figures that follow, when reference is made to two overlapping photographs, this expression is used because of its brevity. Actually, if two photographs were superposed, the overlapping part of one would prevent the observation of points under the other. The superposition is accomplished optically in the plotting machine. The ordinary stereoscope presents a common example of such optical superposition. Futhermore, if photographs are used, the distances ${\overline{N_{1}}}_{1}$ and ${\overline{N_{2} P_{2}}}_{2}$ are principal distances instead of calibrated focal lengths. If the photographs are contact prints from the negatives, and if there is no shrinkage in negative or print, the principal distance of the photograph and calibrated focal length of the camera become identical. The lengths are referred to as calibrated focal lengths because the errors of the camera are being considered and it seems advisable to exclude, so far as convenient, effects arising from the processing of the film or subsequent methods of reproduction. 
$P_{2}$, and $\bar{P}_{1} \bar{N}_{1}$ and $\bar{P}_{2} \bar{N}_{2}$ are the two principal distances, assumed equal to the calibrated focal length. If the photographs are viewed stereoscopically from $N_{1}$ and $N_{2}$, one has a true stereoscopic model, of which the scale factor is $f / a$, where $a$ is the altitude of the camera at the time of the exposure. The point $O$ is the correct position in the model, in accordance with the scale, of a point at an elevation $h$ above the reference plane. It is imaged on photographs 1 and 2 at points $O_{1}$ and $O_{2}$. With the correct value of the calibrated focal length, the two photographs, when mounted in the plotting machine, are viewed from points $N_{1}$ and $N_{2}$, and $O$ is correctly located at the intersection of lines $\overline{N_{1} O_{1}}$ and $\overline{N_{2} O_{2}}$. Because of the error $\Delta f$ in the value of the calibrated focal length, the photographs will be so mounted in the plotting machine that the two photographs are viewed from points $N_{1}^{\prime}$ and $N_{2}^{\prime}$ and the point $O$ is incorrectly located at $O^{\prime}$, the intersection of lines $\overline{N_{1}^{\prime} O_{1}}$ and $\overline{N_{2}^{\prime} O_{2}}$. The displacement $\overline{O O^{\prime}}$ is equal to $\Delta h_{f} f / a$, where $\Delta h_{f}$ is the consequent error in elevation of the point $O^{\prime}$. From similar triangles it is readily seen that

$$
\Delta h_{f}=\frac{h a}{a f} \Delta f=\frac{h}{f} \Delta f .
$$

From the similar triangles it is also apparent that $O^{\prime}$ is directly over $O$. Therefore, if $\Delta l_{f}$ denotes the horizontal displacement arising from the error in the calibrated focal length,

$$
\Delta l_{f}=0 .
$$

In eq 3 , if $\pm \Delta f$ is the probable error of the calibrated focal length, $\pm \Delta h_{f}$ will be the resulting contribution to the probable error of the elevation of a point on the map.

\section{CAUSES OF VARIATION IN CALIBRATED FOCAL LENGTH}

Although the calibrated focal length of a camera may be correctly determined for certain given conditions, it must be remembered that changes in the scale of the negative can arise from various causes such as failure of the film to always occupy the same position in the camera because of poorly designed or poorly constructed devices for removing and replacing the magazine, failure of the lens to return precisely to its original position after it is removed for cleaning, expansion of the camera cone with change of temperature, and other similar causes. All of these can be considered and their effects evaluated as if they were changes in the calibrated focal length arising from changes in the dimensions of the camera.

For a camera with a lens having a focal length of $150 \mathrm{~mm}$ and a cone made of brass or aluminum, it is interesting to note that a change of $50^{\circ} \mathrm{F}$ in temperature will introduce a change in the length of the cone of $0.08 \mathrm{~mm}$. If the photographic film showed tne same percentage change in its dimensions it would largely compensate for the change in the cone and the camera would require little or no correction for temperature. If the cone and film have different coeffi- 
cients of expansion the resulting coefficient for the camera will depend upon their difference. ${ }^{7}$

However, the great uncertainty in the determination of the temperature coefficient and its application lies in the lack of information as to whether or not the camera and its spool of film have time to attain temperature equilibrium during the period required for ascent of the plane and before the exposures are begun. It is fortunate that preliminary considerations indicate that the temperature correction is so small that an approximate estimate of its value should be sufficiently accurate.

\section{DISTORTION}

\section{VARIATION OF DISTORTION WITH SELECTION OF FOCAL LENGTH}

A photographic objective is designed to be symmetrical about its axis and, although the errors of workmanship and assembly necessarily imply departures from this symmetry, it is commonly assumed that the distortion of a photographic objective is also symmetrical about the axis of the lens. In such a case the displacement of any point from its distortion-free position is along a radius, either from or toward the center of the plate. In the presence of distortion no value of $f$ can be found that will satisfy eq 2 for all parts of the negative. Consequently, when distortion is to be considered, it is customary to modify eq 2 by the addition of a correction term, writing it

$$
\tan \beta=\frac{y-\Delta y}{f}
$$

In this equation, $y$ is the measured distance from the principal point to the image point, $\Delta y$ is the linear distortion, and $y-\Delta y$ is the distortion-free position of the image point. The angle $\beta$ is the angular distance of the point from the center of the field.

The distortion $\Delta y$ is not a constant but is different for points in different parts of the field. If it is assumed that the distortion can be satisfactorily represented as symmetrical about the center of the field, it will be the same for all points at a given distance from the center and therefore will be a function of $\beta$ only. In accordance with this assumption, in the reports of the National Bureau of Standards, in the table headed "Distortion" the values of $\Delta y$ are tabulated for intervals of $5^{\circ}$, measured from the center of the plate.

Equation 5 can be written

$$
\Delta y=y-f \tan \beta,
$$

where $f \tan \beta$ is the distance from the center of the plate to the distortion-free position, as given by eq 2 . This equation shows that the distortion-free position $(y-\Delta y)$ is a function of the value of $f$ that has been chosen and that, in consequence, $y$ being fixed in value, $\Delta y$ can be modified at will by changing the value of $f$. After any value of $f$ is chosen, the corresponding values of $\Delta y$ can be obtained from eq 6 , and a consistent description of the image is obtained. For lenses of usual

\footnotetext{
${ }^{7}$ This argument is not rigorously exact because there will be a change in the position of the second nndal point of the lens resulting from the change in the index of refraction of the lens components with tempera. ture. However, in most, if not in all cases, this will be relatively unimportant in comparison with the expansion of the camera cone and flm.
} 
construction there will, in general, be a small range of values of $f$ for which the values of $\Delta y$ vary approximately as the cube of $\tan \beta$ over the field of view that is utilized. When $\Delta y$ varies as $\tan ^{3} \beta$, it follows that its value is relatively small for points in the neighborhood of the center of the field and over this region, therefore, for these values of $f$, eq 2 with no correction term applies with fair accuracy.

When $f$ is so chosen that $\Delta y$ varies approximately as $\tan ^{3} \beta, \Delta y$ has the same sign over the entire field and it has its maximum value at the extreme edge of the field. But values of $f$ can be chosen for which the distortion has one sign for values of $\beta$ less than some given value and the opposite sign for points beyond. With such an adjusted value of $f$ the distortion is distributed more uniformly, so to speak, over the entire negative and never equals in magnitude the value that would exist at the edge of the field if the equivalent focal length of geometric optics were selected.

Either system of values describes the imagery correctly and will lead to the same final result in the map, provided no quantities are neglected when the computations are made. If, however, the plotting machine has no provision for introducing the term $\Delta y$, or if the distortion is only partially compensated by the machine, the two systems of values may be expected to lead to different results. To determine the proper value of $f$ to be selected as the calibrated focal length, it is necessary to consider the method by which a map is constructed.

\section{(a) CRITERION FOR SELECTING THE CALIBRATED FOCAL LENGTH}

When adjusting the negative in a machine an attempt is made to fit certain points on the negative to the corresponding control points that have been carefully surveyed. In the presence of distortion an exact fit cannot be secured. The calibrated focal length should have the value that will lead to the least error in the map as a whole. This requirement does not lead directly to a criterion governing the choice of the calibrated focal length, because the distribution of the control points and the overlap of the photographs govern the manner in which the distortion introduces error into the map. Fortunately the distortion of the modern lens is so small that the different methods of adjusting the focal length lead to results which do not differ greatly. A simple and satisfactory basis for adjustment is afforded if the calibrated focal length is chosen to make the maximum value of the distortion in the photograph as small as possible.

This criterion can be applied by means of eq 6 . If $\Delta y$ of this equation is defined as a function of $\beta$, by differentiation and the usual method of determining a maximum or minimum, one can determine the value of $f$ for which the maximum value of $\Delta y$ within a field of given angular extent is a minimum. To illustrate this, let it be assumed that a lens covers a field having a half-angle of $35^{\circ}$ and that the distortion, referred to the equivalent focal length, varies as the cube of the tangent of the angular distance from the center of the field. If $D$ is the maximum distortion, when referred to the equivalent focal length, then the calibrated focal length will be $1.06 D$ greater than the equivalent focal length. The maximum absolute value of the distortion will be $0.25 D$, and this value will be attained for the values $\beta=19.3^{\circ}$ and $\beta=35^{\circ}$. 
It should perhaps be mentioned that this change from the equivalent to the calibrated focal length in order to vary the distribution of the distortion over the field has no relation to the depth of focus. In this respect it is quite different from an alteration of the back focal length because of the presence of curvature. Such an adjustment was mentioned (section II-1) as possible, because the focal plane can be shifted over an appreciable range without seriously affecting the definition at the center of the field. When, however, the equivalent focal length is changed to compensate for distortion, the position of the focal plane is not altered. The change relates to computations only, the constant of eq 2 being changed in order to obtain a scale factor that better fits the photograph, but no change is made in the distance from camera lens to photographic film.

\section{ERROR ARISING FROM DISTORTION}

After the calibrated focal length has been adjusted to reduce the maximum value of the distortion as much as possible, the effect of the remaining distortion upon the map must be considered. If the maximum value of this residual distortion is $\Delta r$, this will represent the maximum error in the horizontal location of any point lying in the datum plane. The error, in general, will be less than $\Delta r$, not only because the point in question may be located at a position on the photograph where the distortion does not attain this maximum value but also because, when the best possible fit is secured between the control points and the distorted system of image points on the photograph, the adjustment that gives the best fit distributes the distortion and yields a further compensatory action beyond that secured by adjusting the focal length.

If the point under consideration does not lie in the datum plane the determination of the resulting error is more involved. One such case is represented in figure 3 , which is an elevation, $\overline{A B}$ being the trace of the photographs 1 and 2 assumed to be so placed that the common control points coincide. The principal points are at $P_{1}$ and $P_{2}$, and $\bar{P}_{1} N_{1}$ and $\bar{P}_{2} N_{2}$ are the two principal distances assumed equal to the calibrated focal length. If the photographs are viewed stereoscopically from $N_{1}$ and $N_{2}$, one has a true stereoscopic model of which the scale is $f / a$, where $a$ is the altitude of the camera at the time of exposure. The point $O$ is the correct position, in the model, in accordance with this scale, of an elevated point that, as a consequence of the relief parallax, is imaged on photographs 1 and 2 at points $O_{1}$ and $\mathrm{O}_{2}$ when there is no distortion.

Actually, because of the distortion, $O$ is imaged at $O_{1}{ }^{\prime}$ and $O_{2}{ }^{\prime}$. In the present illustration it is assumed that the displacement arising from distortion in each photograph is positive, that is, the point $O_{1}^{\prime}$ is displaced outward from point $P_{1}$, and $O_{2}{ }^{\prime}$ is displaced outward from point $P_{2}$, the two displacements being $\Delta r_{1}$ and $\Delta r_{2}$, respectively. If $O$ were imaged at points $O_{1}$ and $O_{2}$ on the two photographs, observations from points $N_{1}$ and $N_{2}$ upon points $O_{1}$ and $O_{2}$ would correctly locate $O$ at the intersection of lines $\bar{N}_{1} O_{1}$ and $\bar{N}_{2} O_{2}$. Actually, however, the observations are made on points $O_{1}{ }^{\prime}$ and $O_{2}{ }^{\prime}$, and $O$ is erroneously located at $O^{\prime}$. The point $O^{\prime}$ is at the vertex of the triangle $O^{\prime} E G$, of which the base, by similar triangles, is $\left(\Delta r_{1}+\Delta r_{2}\right)(a-h) / a$, and the angles at the two ends of the base may, without important 
error, be assumed to be $90^{\circ}-\beta_{1}$ and $90^{\circ}-\beta_{2}$. (These values differ from the true values by the angles $O_{1} N_{1} O_{1}{ }^{\prime}$ and $O_{2} N_{2} O_{2}{ }^{\prime}$, angles of a few minutes of arc, shown much exaggerated in the drawing.)
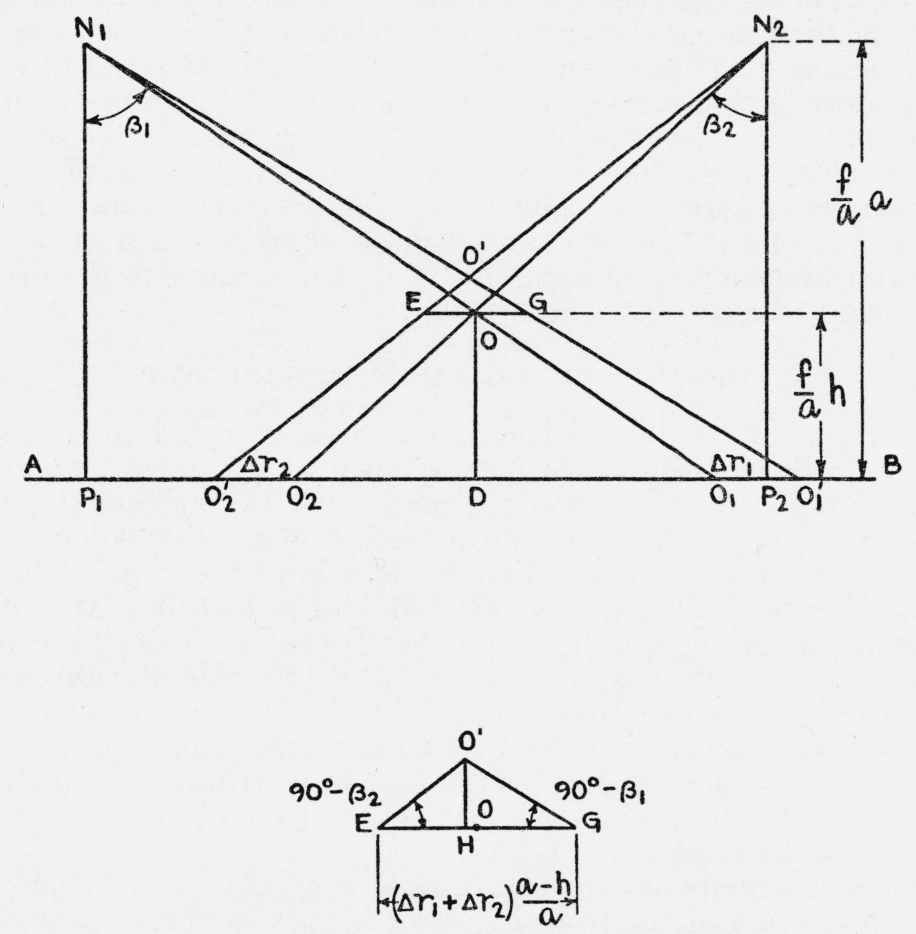

FIGURE 3.-The error arising from distortion.

The elevated point $O$ is imaged at $O_{1}{ }^{\prime}$ and $O_{2}{ }^{\prime}$ instead of at $O_{1}$ and $O_{2}$ because of the distortions $\Delta r_{1}$ and $\Delta r_{2}$. Consequently the point $O$ is erroneously determined to be at $O^{\prime}$, displaced vertically and horizontally from its true position.

For greater clearness, this triangle is shown to a much larger scale, below the main figure. The length $\overline{O^{\prime} H}$ is $\Delta h_{d}^{\prime} f / a$, where $\Delta h_{d}^{\prime}$ is the error in elevation resulting from the presence of distortion. To obtain a convenient expression for $\Delta h_{d}^{\prime}$, one writes

$$
\begin{aligned}
\frac{\overline{E H}}{\overline{H G}}=\frac{\cot E}{\cot G}=\frac{\tan \beta_{2}}{\tan \beta_{1}} & \\
\frac{\overline{E H}+\overline{H G}}{\overline{H G}} & =\frac{\left(\Delta r_{1}+\Delta r_{2}\right)(a-h)}{\overline{H G} a}=\frac{\tan \beta_{1}+\tan \beta_{2}}{\tan \beta_{1}} \\
\overline{H G} & =\frac{\left(\Delta r_{1}+\Delta r_{2}\right) \tan \beta_{1}}{\tan \beta_{1}+\tan \beta_{2}} \frac{(a-h)}{a} \\
\Delta h_{d}{ }^{\prime} \frac{f}{a}=\overline{H G} \cot \beta_{1} & =\frac{\left(\Delta r_{1}+\Delta r_{2}\right)}{\tan \beta_{1}+\tan \beta_{2}} \frac{(a-h)}{a}
\end{aligned}
$$

If $\overline{O_{1} O_{2}}$ (greatly exaggerated in fig. 3 ) is neglected as small in comparison with $\overline{P_{1} P_{2}}$, this equation may be written 


$$
\Delta h_{d}^{\prime}=\frac{\overline{N_{1} P_{1}}(a-h)}{\overline{P_{1} P_{2}} f}\left(\Delta r_{1}+\Delta r_{2}\right) .
$$

In constructing figure 3 , it was assumed that the distortions at $O_{1}$ and $\mathrm{O}_{2}$ are positive in the two photographs. If it should occur that the distortion is positive in one photograph and negative in the other, the quantity $\left(\Delta r_{1}+\Delta r_{2}\right)$ becomes the difference of the absolute values of the two distortions. In all cases the value of $\left(\Delta r_{1}+\Delta r_{2}\right)$ will be less than $2 \Delta r$, where $\Delta r$ is the maximum value of the distortion. Furthermore, the ratio $(a-h) / a$ for any point lying above the datum plane will not exceed 1 . If the ratio $\overline{N_{1} P_{1}} / \overline{P_{1} P_{2}}$ is denoted by $k$, eq 7 may be written

$$
\Delta h_{d}^{\prime} \leq \frac{2 k a}{f} \Delta r .
$$

This inequality will be true for all points that lie above the datum plane.

The ratio $\bar{N}_{1} P_{1} / \bar{P}_{1} P_{2}$ is equal to the ratio of camera altitude to stereoscopic base and is a function of the focal length of the camera objective and the amount of overlap. The values for a film 9 by 9 inches and for different percentages of overlap are given in table 2 .

TABLE 2.-Values of $k\left(\right.$ or $\left.k^{\prime}\right)$, the ratio of altitude to stereoscopic base for different percentages of overlap

\begin{tabular}{|c|c|c|c|}
\hline \multirow{2}{*}{$\begin{array}{c}\text { Percentage } \\
\text { of overlap }\end{array}$} & \multicolumn{2}{|c|}{ Focal length of objective } \\
\cline { 2 - 3 } & 6 in. & 7 in. & 8 in. \\
\hline & & & \\
10 & 0.74 & 0.86 & 0.99 \\
20 & .83 & .97 & 1.11 \\
30 & .95 & 1.11 & 1.27 \\
40 & 1.11 & 1.29 & 1.48 \\
50 & 1.33 & 1.54 & 1.77 \\
60 & 1.66 & 1.95 & 2.22 \\
70 & 2.22 & 2.59 & 2.96 \\
80 & 3.34 & 3.88 & 4.44 \\
90 & 6.66 & 7.77 & 8.89 \\
\hline
\end{tabular}

In a flight strip the customary overlap is 60 percent. The value of $k$ for an overlap of this amount or less does not exceed 1.7, 2.0, and 2.2 , for camera objectives with focal lengths of 6,7 , and 8 inches, respectively. This illustrates the advantages of the lens of shorter focal length, for a given scale factor, $f / a$, in minimizing the error in elevation that arises from the presence of distortion.

The horizontal displacement arising from the presence of distortion will be denoted by $\Delta l^{\prime}{ }_{d}$. Referring to figure 3 , it is evident that a vertical through $\mathrm{O}^{\prime}$ will always cut $\overline{E G}$, the base of the triangle $O^{\prime} E G$. Furthermore, the length $\overline{O E}$ or $\overline{O G}$ will never exceed $(a-h) \Delta r / a$. Therefore,

$$
\Delta l_{d}^{\prime} \leqq \Delta r .
$$

In eq 8 and $9, \Delta r$ is the maximum distortion on a photograph, and, consequently, $\Delta h^{\prime}{ }_{d}$ and $\Delta l^{\prime}{ }_{d}$ are the maximum errors resulting from the distortion. It is convenient to have these equations in a form 
suitable for dealing statistically with the resulting errors of the map. The distortion in a photograph assumes all values from $-\Delta r$ to $+\Delta r$, with the maximum values in two zones, one near the edge of the field, the other at an intermediate distance. For a given object point, the distortion on the two photographs will, in general, be different, because the images will not fall in homologous positions on the two photographs. Given the distortion measurements for a lens, it is possible to compute the error of location and elevation for any given object point. This error will vary with the elevation of the object point as well as with the projected position of the point upon the datum plane and will range in value from the maximum positive value, through zero, to maximum values in the opposite sense, the maximum error occurring only when the distortion for the images of a given object point has its maximum value in each of the two photographs that are combined to produce a stereoscopic model. In a general discussion of this nature it is not possible to consider these errors independently, but they must be dealt with by a statistical method.

For errors that are truly "accidental" and follow the normal frequency curve, the error whose probability is one in one hundred, is approximately four times the probable error. Consequently, if the magnitudes of the errors resulting from distortion followed such a distribution law the probable error, the error that is equal to or larger than the errors for 50 percent of the points, could be assumed, with satisfactory precision, to be one fourth the maximum error that has just been deduced. Actually, however, the errors arising from distortion are systematic and this is evidenced by the regular distribution of the errors over the photograph. A typical distortion curve for a lens has been assumed and, for a large number of points, the resulting horizontal and vertical errors arising from distortion have been computed. A study of these errors indicates that 50 percent of the errors may be expected to be less than or equal to 0.4 of the maximum error. Accordingly, in order to treat the errors arising from distortion in the same manner as those arising from variation of calibrated focal length or location of principal point, the maxımum error arising from distortion, multiplied by 0.4 , will be considered to be a measure of the error arising from distortion and will be applied in the same manner as a probable error. This error is analogous to the probable error in that it is a "50-percent error" but differs from it in that it relates to errors that are not "accidental" in origin.

Accordingly,

$$
\begin{aligned}
\Delta h_{d} & \leqq 0.8 \frac{k a}{f} \Delta r \\
\Delta l_{d} & \leqq 0.4 \Delta r
\end{aligned}
$$

where $\pm \Delta h_{d}$ and $\pm \Delta l_{d}$ are contributions made by the distortion to the probable errors of elevation and horizontal displacement of a point on the map.

It should be noted that eq 10 has the reciprocal of the scale factor $f / a$ in the right-hand member and $\pm \Delta h_{d}$, consequently, is the probable error of the elevation. Equation 11, on the other hand, gives probable error as a displacement on the photograph or on a map having the same scale as the photograph. These are the convenient forms of these equations because of the manner in which the allowable errors of 
a map are commonly specified. If one wishes to obtain an inequality similar to that of eq 11 giving the error on a map having a scale different from that of the photograph, the right-hand member must be divided by the scale factor of the photograph and be multiplied by the scale factor of the map.

\section{VALUES OF FOCAL LENGTH AND DISTORTION REPORTED FOR A LENS NOT MOUNTED IN A CAMERA}

In the reports of the National Bureau of Standards on lenses submitted for test unassembled in cameras the equivalent focal length is given rather than an adjusted focal length. Several advantages result from this. Lenses are frequently submitted for test before acceptance and, when the distortions of all lenses are referred to the paraxial value of the focal length, the values permit a more direct comparison of different lenses, because no question arises as to whether or not the difference in distortion may be the consequence of a better choice of focal length rather than of greater freedom from distortion. In many cases, when a large number of lenses have been submitted for test at the same time, a measurement of the distortion at a single point, usually $30^{\circ}$ from the axis, has been considered sufficient to classify a lens. In such cases some convention, of which the choice of the paraxial value of the focal length seems to be the simplest, is particularly necessary, as otherwise, by a modification of the value of the focal length, the distortion for the one point could be made to have any desired value. The measurement of the distortion in this manner also permits direct comparison of the values with the computed values of distortion, as given and plotted in many publications dealing with photographic objectives. ${ }^{8}$

\section{PRINCIPAL POINT OF THE FOCAL PLANE}

\section{INDEX MARKS FOR PRINCIPAL POINT}

Mapping cameras utilize various devices for locating the principal or collimation point. When a glass pressure plate is employed, a cross may be etched on the side of the plate in contact with the film to register on the negative at the time of the exposure. When a pressure plate is not used, four projections in the focal plane are placed so that they are registered on the negative and are so located that lines joining the two pairs of opposite points determine the position of the principal point by their intersection. Sometimes the four index marks are registered on the film by four small optical projectors attached to the interior of the camera cone, this method giving sharper and finer lines than can be obtained by the simpler silhouette.

\section{RELATION BETWEEN THE PRINCIPAL POINT AND THE AXIS OF THE LENS}

It has already been mentioned that the principal point is located at the foot of the perpendicular from the center of perspective to the focal plane, and that this will not correspond with the intersection of the focal plane and the axis of the lens, if the lens is tipped from its

\footnotetext{
'V. Rohr, Theorie und Geschichte des Photographischen Objektivs (Julius Springer, Berlin, 1899). Handbuch der Wissenschaftlichen und Angewandten Photographie, vol. I, Das Photographische Objektiv (Julius Springer, Wien, 1932).
} 
normal position. The errors resulting from such a tipping of the lens are not as serious as is perhaps commonly considered. In most cases the only error introduced by such a maladjustment arises from the possible blurring of the image because the plane of best focus is inclined with respect to the plane of the film. If there is a great deal of distortion a further error may be introduced because the distortion will not be symmetrical about the principal point.

\section{ERROR ARISING FROM INCORRECT LOCATION OF THE PRINCIPAL POINT}

When adjusting a negative in a plotting machine an error in the location of the principal point, as registered on the negative, causes the negative to be located in the machine in a position displaced in its own plane from the true position. This is illustrated in figure 4. The drawing on the left is assumed to illustrate the actual condition at the time of the exposure. The true principal point is at $O^{\prime}$. The image of $O$, the point directly below the camera, coincides with the principal point. If the camera is assumed to be incorrectly calibrated, so that $C^{\prime}$ is indicated as the principal point, the final adjustment in the plotting machine leads to the implicit conclusion that the camera was vertically over the point $C$ at the time of the exposure. This corresponds to a displacement of the camera from its actual position through the horizontal distance $\Delta c a / f$, where $\Delta c$ is equal to the distance $\overline{O^{\prime} C^{\prime}}$, the error in the location of the principal point. As long as the camera is

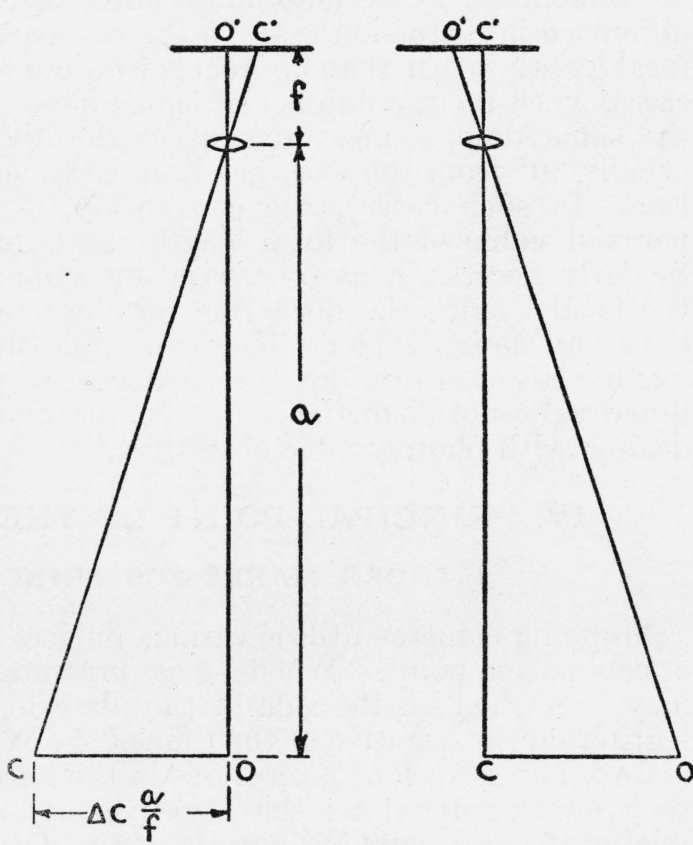

FIGURE 4.-The error in the assumed position of the camera arising from an incorrect position of the principal point.

The exposure was made with the camera above 0 , but it is assumed to have been above $C$ because of the error in the indicated location of the principal point.

vertical and the terrain plane and horizontal, the photographs taken with the camera in the two positions will be identical except for the displacement of each point of the picture through the distance $\overline{C^{\prime} O^{\prime}}$, a characteristic that has no bearing upon the accuracy of the map. Since the two negatives are identical the control points, if lying in the reference plane, can be fitted accurately when $O^{\prime}, C^{\prime}$, or any other point is assumed to be the principal point. Therefore, just as an error in the focal length introduces no error when the terrain is plane 
and horizontal, so an error in the location of the principal point introduces no error under similar simplified assumptions.

(a) CASE IN WHICH THE TWO OVERLAPPING PHOTOGRAPHS ARE FROM A SINGLE FLIGHT STRIP

The region to be mapped usually has depressions and elevations which produce relief parallax. In order to determine the error that results from an incorrectly located principal point, it is necessary to consider the interpretation made from two photographs that serve as members of a stereoscopic pair when the map is being made. If these photographs are successive photographs from a flight, it may be assumed, without serious error, that the camera underwent no rotation in azimuth between the two exposures. Consequently, not
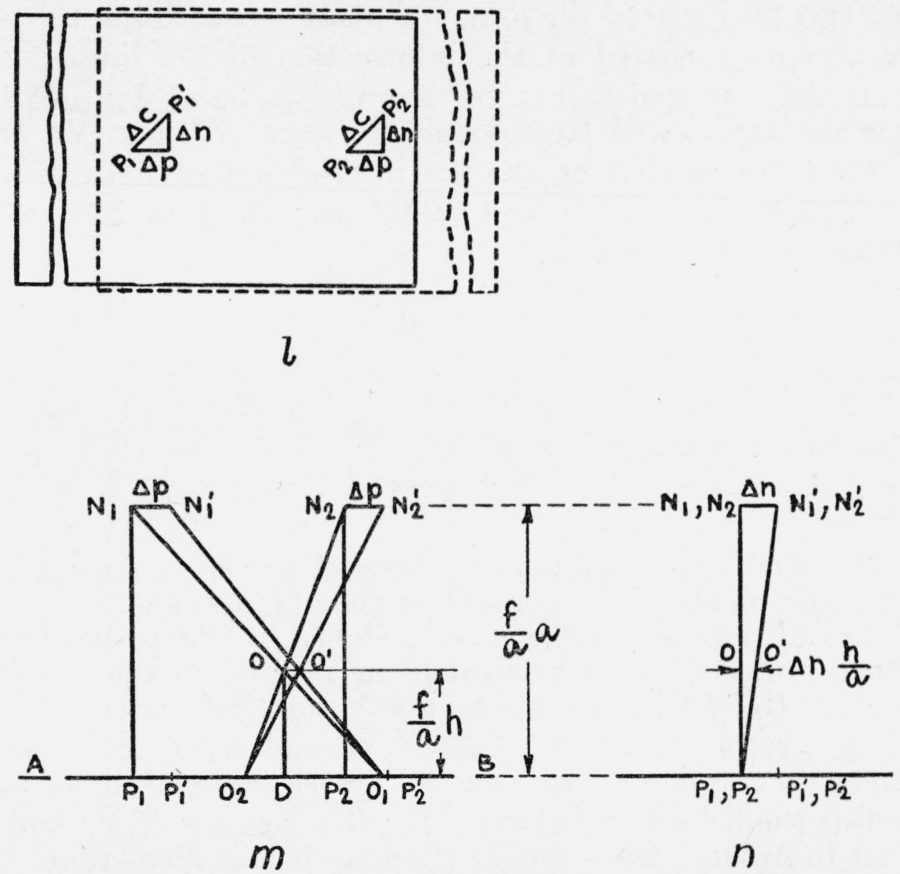

FIGURE 5.-The error arising from an incorrectly located principal point.

When the two overlapping photographs are selected from a single flight-strip, the displacements of the principal points, denoted by $\triangle c$, are similarly oriented in the two photographs and the elevated point at $O$ is incorrectly located at $O^{\prime}$, a point displaced horizontally but having the correct elevation. Diagrams $m$ and $n$ are projections. A projected point that corresponds to two or more original points is designated by the symbols of the several points, separated by commas.

only the magnitude of the displacement of the principal point, but also the azimuth of a line joining the true principal point with the assumed principal point will have the same value for the two exposures. This is illustrated in figure 5 (l) which is a diagrammatic representation of the two photographs with the overlapping portions superposed to bring the common control points (in the datum plane) into coincidence. The two principal points are at $P_{1}$ and $P_{2}$, the assumed incorrect principal points are at $P^{\prime}{ }_{1}$ and $P^{\prime}{ }_{2}$. The displacements, 
$\Delta c$, may be resolved into components $\Delta p$ and $\Delta n$, parallel and normal to the stereoscopic base, $\bar{P}_{1} P_{2}$.

Figure $5(\mathrm{~m})$ is an elevation in which significant points are projected on a vertical plane parallel to the stereoscopic base. $\overline{A B}$ is the trace of the plane of the photographs, and lines $\bar{P}_{1} N_{1}$ and $\bar{P}_{2} N_{2}$ represent the principal distances of the two photographs, assumed equal to the calibrated focal length. If the photographs are viewed stereoscopically from $N_{1}$ and $N_{2}$ one has a true stereoscopic model, of which the scale is $f / a$, where $a$ is the altitude of the camera at the time of exposure. The point $O$ is the position, in the model, in accordance with this scale, of an elevated point that, because of relief parallax, is imaged at $O_{1}$ and $O_{2}$ in the two photographs.

When these two photographs are mounted in a mapping machine, if the correct positions of the principal points are utilized, the point $O$ will be correctly located at the intersection of the lines ${\overline{N_{1} O_{1}}}_{1}$ and $\bar{N}_{2} \mathrm{O}_{2}$. If the principal points are assumed to be at $P_{1}^{\prime}$ and $P_{2}^{\prime}$ the photographs are viewed stereoscopically from $N_{1}{ }^{\prime}$ and $N_{2}^{\prime}$ and the

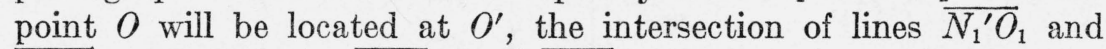
${\overline{N_{2}{ }^{\prime} O_{2}}}_{\text {. The lengths }}{\overline{N_{1} N_{1}}}^{\prime}$ and ${\overrightarrow{N_{2} N_{2}}}^{\prime}$ are equal to $\Delta p$, and from similar triangles it is seen that

$$
\Delta h_{p}=0,
$$

where $\Delta h_{p}$ is the error in elevation, and

$$
\Delta l_{p}=\frac{h}{a} \Delta p,
$$

where $\Delta l_{p}=O O^{\prime}$ is the component, parallel to the stereoscopic base, of the displacements of the point $O$ on the photographs.

In figure $5(\mathrm{n})$ a second elevation is shown in which the significant points are projected on a vertical plane normal to the stereoscopic base. With this projection the points $N_{1}$ and $N_{1}{ }^{\prime}$ are superposed on $N_{2}$ and $N_{2}{ }^{\prime}$, respectively, and lines ${\overline{N_{1}{ }^{\prime} O_{1}}}_{1}$ and ${\overline{N_{2}{ }^{\prime} O_{2}}}_{2}$ are superposed, appearing as a single line. These two superposed lines actually intersect at the height $h f / a$ above $A B$. The lengths $\bar{N}_{1} N_{1}^{\prime}$ and $\bar{N}_{2} N_{2}^{\prime}$ are equal to $\Delta n$ and, from similar triangles it is evident that

$$
\Delta l_{n}=\frac{h}{a} \Delta n,
$$

where $\Delta l_{n}=\overline{O O^{\prime}}$ is the component, normal to the stereoscopic base, of the displacements of the point $O$ on the photographs. The component $\Delta n$, like $\Delta p$, produces no error in elevation.

From eq 13 and 14 it follows that

$$
\Delta l_{c}=\frac{h}{a} \Delta c,
$$

where $\Delta l_{c}$ may be considered to be the vector displacement of the point $O$ from its true position resulting from the vector displacement 
$\Delta c$ of the assumed position of the principal point from its true position. These are actual displacements measured on the stereoscopic model constructed from the photographs.

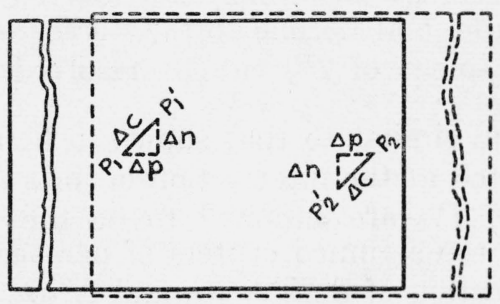

$\imath$

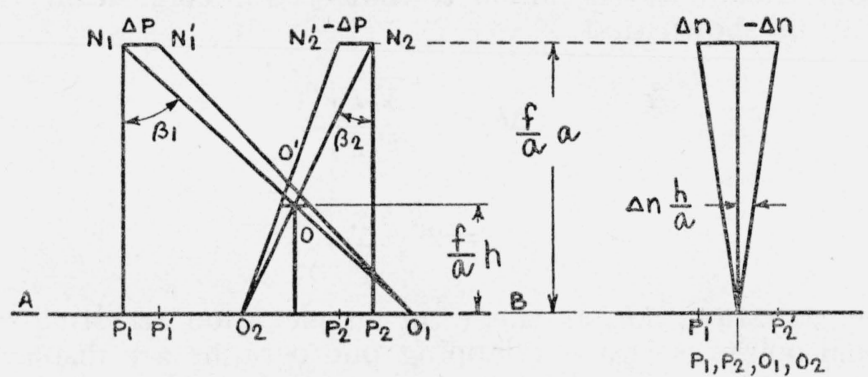

$m$

$n$

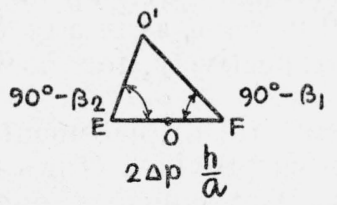

FIGURE 6.-The error arising from an incorrectly located principal point.

When the two overlapping photographs are selected from two adjacent flight-strips flown in opposite directions, the displacements of the principal points, denoted by $\Delta c$, differ in azimuth $180^{\circ}$, and the elevated point at $O$ is incorrectly located at $O^{\prime}$, the elevation and the horizontal position both being in error. Diagrams $m$ and $n$ are projections. A projected point that corresponds to two or more original points is designated by the symbols of the several points, separated by commas.

(b) CASE IN WHICH THE TWO OVERLAPPING PHOTOGRAPHS ARE FROM ADJACENT FLIGHT STRIPS

If the two pictures under stereoscopic examination are overlapping photographs from two adjacent flight strips, the plane will have been flying, in most instances, down one strip and up the other. Consesequently, the azimuths of the displacements of the principal points from their true positions in the two photographs will differ by $180^{\circ}$. This is illustrated in figure $6(1)$, which is a diagrammatic representation of the two photographs with the overlapping portions superposed to bring the common control points (assumed to lie in the datum plane) into coincidence. As in figure 5 , the two principal points are at $P_{1}$ and 
$P_{2}$, the assumed principal points at $P_{1}^{\prime}$ and $P_{2}^{\prime}$. The vector displacements of the points $P^{\prime}{ }_{1}$ and $P^{\prime}{ }_{2}$ from $P_{1}$ and $P_{2}$ are $\Delta c$ and $-\Delta c$, respectively, the negative sign resulting from the $180^{\circ}$ difference in sign of the two values. The displacement of $P_{1}^{\prime}$ may be resolved into the components $\Delta p$ and $\Delta n$, parallel and normal to the stereoscopic base, $\bar{P}_{1} P_{2}$. Similarly, the displacement of $P_{2}^{\prime}$ can be resolved into the components $-\Delta p$ and $-\Delta n$.

Figure $6(\mathrm{~m})$ is an elevation, similar to that shown in figure $5(\mathrm{~m})$. The point $O$ is correctly located at the intersection of lines $\overline{N_{1} O_{1}}$ and $\bar{N}_{2} O_{2}$. When points $P_{1}^{\prime}$ and $P^{\prime}{ }_{2}$ are assumed to be the principal points, $N^{\prime}{ }_{1}$ and $N^{\prime}$ becomes the assumed centers of perspective and $O$ is located at $O^{\prime}$, the intersection of lines ${\overline{N^{\prime}}}_{1} \mathrm{O}_{1}$ and ${\overline{N^{\prime}}}_{2} \mathrm{O}_{2}$. Also it is evident from the figure that $O^{\prime}$ will, in general, be displaced from its true position both in vertical and horizontal directions. The point $O^{\prime}$ is at the upper vertex of a small triangle with the base horizontal and point $O$ located at its midpoint. The length of the base is $2 \Delta p h / a$. By geometrical analysis similar to that used in connection with figure 3 , it can be shown that

$$
\begin{aligned}
\Delta h_{p} & =2 \frac{h \overline{N_{1} P_{1}}}{f \overline{P_{1} P_{2}}} \Delta p \\
& =2 \frac{k^{\prime} h}{f} \Delta p .
\end{aligned}
$$

In this equation, $\Delta h_{p}$ is the error in elevation resulting when the principal points of two overlapping photographs are displaced a distance, $\Delta p$, in opposite directions along the stereoscopic base. Also $k^{\prime}$ is the ratio $\bar{N}_{1} P_{1} / \bar{P}_{1} \bar{P}_{2}$, as tabulated in table, 1 , and is distinguished from $k$ of eq 10 because the customary overlap for successive flight strips is 30 percent instead of 60 percent, as in a given strip. Values of $k^{\prime}$ are $0.95,1.11$, and 1.27 , respectively, for focal lengths of 6,7 , and 8 inches.

In the triangle $E O^{\prime} F$ the horizontal displacement of the point $O^{\prime}$ will be less than one-half the base, provided $O$ lies vertically over a point falling between $P_{1}$ and $P_{2}$. Consequently, one may write

$$
\Delta l_{p} \leqslant \frac{h}{a} \Delta p
$$

where $\Delta l_{p}$ represents the actual displacement of $O^{\prime}$ from $O$, as measured on the photograph.

Figure $6(\mathrm{n})$ is an elevation in which the significant points are projected on a plane normal to the stereoscopic base. Unlike figure $5(\mathrm{n})$, the components $\Delta n$ and $-\Delta n$ are here turned in opposite directions. The lines $\overline{N_{1}^{\prime} O_{1}}$ and $\overline{N_{2}^{\prime} O_{2}}$ do not intersect at all (their projections intersect in figure 6(n), but at this point the lines themselves are separated by the distance $\overline{\mathrm{O}_{1} \mathrm{O}_{2}}$ ). One line lies on one side of the vertical plane containing the stereoscopic base (the trace of this plane is $\overline{N_{1} O_{1}}$ ) and the other line lies on the other side. The point of nearest approach for these two lines has approximately the same elevation as the point $O^{\prime}$ in figure $6(\mathrm{~m})$. It is evident from this figure, where $\Delta n$ and $-\Delta n$ are shown greatly exaggerated, that strict stereoscopic fusion cannot 
be attained for this condition, but it is attainable in satisfactory degree even though the two fields in the viewing instrument will be at slightly different heights. Reference to figure $6(n)$ shows that neither line departs from the correct position for the intersection more than $\Delta n h / a$, and it appears reasonable to assume that the horizontal position of the point will be obtained with a horizontal error not exceeding this amount. The components $\Delta n$ and $-\Delta n$ do not introduce any significant error in the elevation.

(c) EQUATIONS GENERALIZED TO APPLY TO THE TWO PRECEDING CASES (a) AND (b)

A consideration of eq 12 and 15 of case (a) and eq 16 and 17 of case (b) shows that one may write

$$
\begin{aligned}
& \Delta h_{c} \leqslant 2 \frac{k^{\prime} h}{f} \Delta c \\
& \Delta l_{c} \leqslant \frac{h}{a} \Delta c .
\end{aligned}
$$

These equations are applicable to either case (a) or case (b) and cover all errors arising from incorrectly located principal points. In eq 18 and $19, \Delta h_{c}$ and $\Delta l_{c}$ are the errors of elevation and horizontal displacement corresponding to the error $\Delta c$ in the location of the principal point. The constant $k^{\prime}$ is taken from table 1 , the overlap being that for adjacent flight strips. As for the previous pairs of equations, eq 18 gives the actual error in elevation and eq 19 gives the displacement as measured on the photograph.

In eq 18 and 19, if $\pm \Delta c$ is the probable error of the location of the principal point, $\pm \Delta h_{c}$ will be the resulting contribution to the probable errors of the elevation and $\pm \Delta c$ the contribution to the probable error of the location of a point on the photograph.

\section{DEPARTURE OF FILM FROM THE FOCAL PLANE}

Photographic film, rather than plates, is commonly used for photogrammetric purposes and the assumption, in all the foregoing discussion, has been that the sensitive photographic emulsion lies in a plane. A suction or pressure back, or a pressure plate is commonly used to flatten the film. If this does not operate perfectly, it is to be expected that the film will be concave toward the objective, and it seems probable that the curvature will be different in the direction in which the film is rolled and in a direction at right angles to it. If a portion of the film is in advance of or behind the position that it should occupy, the image falling upon this portion of the film will be displaced the distance $\Delta d \tan \beta$ toward or from the center of the negative, when $\Delta d$ is assumed to be the displacement of the film. If the film is curved in an approximately symmetrical manner from the focal plane there will be a systematic displacement over the field somewhat resembling that arising from distortion, and it is a reasonable assumption that this will be a maximum at the edge of the field. If the curvature of the film were quantitatively known and sufficiently uniform from exposure to exposure, a change in the value of the calibrated focal length could be made in order to secure compensation, as was done for distortion. For distortion, with a field of view of $60^{\circ}$, the correction 
to the calibrated focal length is approximately equal to the distortion at the edge of the field referred to the paraxial focal length, and, as a first assumption, it appears that a somewhat similar relation might exist between a regular departure of the emulsion from a plane and the change in the calibrated focal length to compensate for it. If, therefore, it is assumed that the film curves from the nominal plane surface by $0.1 \mathrm{~mm}$, the resulting error is approximately the same as would result from an error of $0.1 \mathrm{~mm}$ in the calibrated focal length, but it is not the same in all parts of the field and cannot be readily compensated.

By decreasing focal length and film size one can increase the expectancy of obtaining planeness of film, but unfortunately the error introduced by a given departure from planeness increases with decrease of focal length, so that exact information regarding the manner in which the departure from planeness diminishes with size of the film is necessary before it can be said whether or not reduction of focal length is advantageous in respect to the error arising from curvature of film. It is important to note that when a 9-by 9-inch film is retained and the angular field increased by the use of a lens of shorter focal length, the planeness of the film must be improved if the error from this source is not to increase.

All of these considerations indicate that the planeness of the film at the time of exposure is an important requirement of the precision camera and that any increase in the accuracy of the precision camera may require a more complicated or better designed device for holding the film flat. This immediately suggests the use of plates rather than films in the photogrammetric camera. However, plates as commercially manufactured are not plane, and, in many cases, the holders are of such a nature that the plate is bent by the springs that serve to retain them in the focal plane. Arnulf and Perrin ${ }^{9}$ have made measurements on photographic plates and report departures from planeness as great as $0.26 \mathrm{~mm}$ when the plate is in the plateholder of an airplane camera. P. Lafouasse ${ }^{10}$ presents data indicating that the films measured by him were somewhat more nearly plane than the photographic plates that he measured. It is probably advisable that additional quantitative data be secured regarding the planeness of films and plates in the types of airplane cameras that are used in this country, and the curvature of the film or plate may be one of the important factors setting a limit to the precision that can be obtained with the photogrammetric cameras now in use.

\section{TYPES OF SHUTTERS}

\section{BETWEEN-THE-LENS SHUTTER}

The type of shutter having its leaves mounted within the lens and between the components, is the one most favored for airplane photography. With this type of shutter all portions of the film are exposed at the same time. Consequently, if the duration of the exposure is too great, with respect to the translatory or angular velocity of the plane, the resulting negative may lack definition because of the movement of the plane, but in no case will there be distortion analogous to that to be considered later in connection with the focal-plane shutter.

Arch. Int. Photogrammetry 7, 2d part, 202 (1930-31).

10 Bul. soc. franç. phot. 24, 29-35(1937). 
The fundamental requirement for a between-the-lens shutter is that it should open as rapidly as possible to its maximum opening, remain open for a given period of time, and then close in the shortest possible time. An ideal shutter would consume zero time in its opening and closing. In such a case the exposure may be considered equal to the product of the area of the shutter aperture by the elapsed time from the opening to the closing of the shutter. During the actual performance of a shutter one does not have the aperture of the shutter completely open during the entire period that elapses between the beginning of the opening and the end of the closing of the shutter. Consequently, one obtains the effective exposure by integrating the area of the varying shutter opening with respect to the time, the integration being extended to cover the over-all length of the exposure. The ratio of the effective exposure to the ideal exposure is defined as the efficiency of the shutter. It is evident that the efficiency of the shutter, in general, will decrease as the exposure is shortened because a proportionately greater time is consumed in opening and closing. It is also evident that the efficiency of a given shutter will increase as a lens is stopped down because, with the smaller diaphragm, the shutter requires less time to open to the full extent of the smaller diaphragm. The motion of the plane sets a limit to the maximum duration of an exposure that can be made without blurring, and a high efficiency is important because it permits more light to reach the film for an exposure of given duration. Lutz and Doyle ${ }^{11}$ describe a shutter with an efficiency of 93 percent for a one-fiftieth second exposure and 80 percent for an exposure of one one-hundred-fiftieth second.

It is important that successive exposures for a given setting of the speed control should be identical, and desirable that the actual speeds do not depart greatly from the nominal speeds. For a precise camera the shutter should be so designed that the lenses can be removed for cleaning or disassembly of the shutter and replaced without necessitating a recalibration of the camera. Some users of cameras desire a shutter sufficiently simple and robust to be repaired in the field and others consider that a shutter should give the minimum of trouble, but that it is desirable to permit no alteration or adjustment of the shutter except when it is returned to the home office.

\section{FOCAL-PLANE SHUTTER}

(a) EFFICIENCY

The focal-plane shutter consists of an opaque blind or curtain containing a transverse slit that travels across the face of the photographic film, approximately in the focal plane. The light reaches the film through the slit and the exposure can be varied by altering the velocity of travel or varying the width of the slit. Adhering to the usual assumptions of geometric optics, the cone of rays proceeding from an object point has its vertex at the film. Therefore, a section of the light cone lying in the focal plane shrinks to a point, and if the curtain cuts the cone in this plane, the cone is entirely intercepted by the curtain or entirely transmitted by the slit at any given instant. Consequently, any small area of the film is illuminated by the entire aperture of the lens during the entire period of the exposure and no time

\footnotetext{
11 Photogrammetric Engineering 3, No. 3, 1-6 (1937).
} 
is lost by the process of opening or closing the shutter. Therefore, if the traveling slit could be placed exactly in the focal plane the efficiency of the shutter would be 100 percent and this type of shutter would be realized in its ideal form.

Obviously, however, it is impracticable to place the curtain of the shutter in the focal plane and it is not unusual to find it a centimeter or more from the surface of the film. In this case the section of the cone of light in the plane of the curtain is a circle of appreciable diameter and the aperture of the lens is not entirely unobstructed until the curtain has travelled a distance equal to the diameter of this section. Consequently, the efficiency of the shutter in its practicable form is not 100 percent and, in important cases, may be quite as low as for the between-the-lens type of shutter. The efficiency of the shutter decreases as the section of the cone of light in the plane of the curtain increases, and this increases as the aperture of the lens is increased or as the distance from focal plane to plane of shutter curtain increases. For the shorter exposures, for which the slit width is narrow, the time required for uncovering the entire aperture of the lens is greater with respect to the total time of exposure than for a wider slit. Consequently, the departure from ideal efficiency is greater for the narrower slit widths. For typical values, the efficiency of the focal plane shutter as a function of the slit width, lens aperture, and distance of curtain from film is given in table 3 .

TABLE 3.-Efficiency of focal-plane shutter

\begin{tabular}{|c|c|c|c|c|c|c|c|c|c|}
\hline \multirow{2}{*}{$\begin{array}{l}\text { Distance from curtain to } \\
\text { focal plane }\end{array}$} & \multicolumn{3}{|c|}{$\begin{array}{l}\text { Width of slit, in milli- } \\
\text { meters, with } f / 4 \text { aper- } \\
\text { ture }\end{array}$} & \multicolumn{3}{|c|}{$\begin{array}{l}\text { Width of slit, in milli- } \\
\text { meters, with } f / 8 \text { aper- } \\
\text { ture }\end{array}$} & \multicolumn{3}{|c|}{$\begin{array}{l}\text { Width of slit, in milli- } \\
\text { meters, with } f / 16 \text { aper- } \\
\text { ture }\end{array}$} \\
\hline & 10 & 20 & 30 & 10 & 20 & 30 & 10 & 20 & 30 \\
\hline $\begin{array}{c}\mathrm{mm} \\
10 \\
20 \\
30\end{array}$ & $\begin{array}{l}\% \\
80 \\
67 \\
57\end{array}$ & $\begin{array}{l}\% \\
89 \\
80 \\
73\end{array}$ & $\begin{array}{r}\% \\
92 \\
86 \\
80\end{array}$ & \begin{tabular}{l|}
$\%$ \\
89 \\
80 \\
73
\end{tabular} & $\begin{array}{r}\% \\
94 \\
89 \\
84\end{array}$ & $\begin{array}{l}\% \\
96 \\
92 \\
89\end{array}$ & $\begin{array}{r}\%_{94} \\
89 \\
84\end{array}$ & $\begin{array}{l}\% \\
97 \\
94 \\
91\end{array}$ & $\begin{array}{r}\% \\
98 \\
96 \\
94\end{array}$ \\
\hline
\end{tabular}

A focal-plane shutter with a $20-\mathrm{mm}$ slit, $20 \mathrm{~mm}$ from the focal plane, may be considered as typical of modern practice for shutters on airplane cameras. For such a shutter the efficiencies for lens apertures of $f / 4, f / 8$, and $f / 16$ are shown in table 3 to be 80,89 , and 94 percent, respectively.

\section{(b) DISTORTION INTRODUCED BY FOCAL-PLANE SHUTTER}

In the preceding section the efficiency has been expressed as the ratio of the actual exposure to the exposure obtained with an ideal shutter having the same over-all duration of exposure for a given point on the photographic film. This over-all duration of the exposure, for a given movement of the airplane, has a maximum value that cannot be exceeded without the presence of blur and loss of definition resulting from the movement of the plane. With the focal-plane shutter, unlike the between-the-lens shutter, different portions of the film are exposed successively as the slit travels across the field of the lens. Consequently, although the exposure for a restricted area of the film is sufficiently short to "stop" the motion and eliminate blur, it is still possible that the plane may move appreciably before the time 
required for exposing the entire area of the film has elapsed. If this is the case, different parts of the photograph are made from different positions in space and the photograph is distorted.

The typical values for a focal plane shutter that were used in the preceding section will again be considered. If the slit is $20 \mathrm{~mm}$ wide and the exposure is $1 / 75$ th second, the curtain travels with a speed of 1,500 mm per second. The direction of travel is the short dimension of the photograph, and it therefore requires somewhat more than a tenth of a second for the curtain to travel $175 \mathrm{~mm}$ (7 in.), the width across the picture. If the plane is traveling at a speed of 200 miles per hour, it travels nearly 30 feet in a tenth of a second. Consequently, the extreme edges of the film are exposed from positions 30 feet apart. There is no true center of perspective for a picture taken under these conditions, because the portion of the negative exposed by the initial movement of the shutter curtain has a center of perspective 30 feet distant from that corresponding to the portion of the negative exposed as the shutter slit left the field of view. If the focal length of the lens is 6 inches and the altitude of the plane is 15,000 feet, this corresponds to a progressive displacement of the principal point of $0.3 \mathrm{~mm}$ on the photograph. Even if the curtain speed is increased so that the same slit width gives a speed of $1 / 225$ th second, the displacement of the principal point for different parts of the negative is $0.1 \mathrm{~mm}$.

A similar displacement of the principal point will also arise from the angular velocity (rolling or pitching) of the airplane. Estimates, based on several intervals of 30 seconds each in smooth air, indicate a value of $1 / 8^{\circ}$ per second (0.002 radian per second) as the maximum value that was encountered, although large variations from this value may be anticipated for different types of planes and for different pilots. With this value of the angular velocity, if the elapsed time for an exposure is 0.1 second, the airplane will swing through an angle of 0.0002 radian during its progress. The corresponding displacement of the principal point for a lens with a focal length of $150 \mathrm{~mm}$ is 0.03 $\mathrm{mm}$. This indicates that the distortion arising from the angular velocity of the plane will not be as great as that arising from the velocity of translation when one is flying over smooth and uniform terrain. When an airplane is flying over nonuniform territory, where upward air currents and bumpiness result, angular accelerations exceeding these values are probable and may introduce serious and unpredictable amounts of distortion in the photographs.

If the direction of travel of the curtain shutter is parallel to the direction in which the airplane moves, it will introduce a distortion consisting of a lengthening or shortening of the picture in the direction of travel. If the curtain travels in a direction at right angles to the motion of the airplane, the distortion will be of a different character in which a square is photographed as a rhombus.

The large ratio of the time that elapses during the exposure of the entire picture to the length of exposure for a given small area of the picture is the fundamental disadvantage of the focal-plane shutter. This ratio decreases as the width of the slit is increased but, with the wider slit, it is necessary to correspondingly increase the velocity of travel of the curtain, a matter of some difficulty, if a short exposure is to be secured. The ratio of total elapsed time to exposure time for a series of slit widths follows. In this tabulation it has been assumed that the film is 7 by 9 inches and that the direction of travel of the 
slit is parallel to the short dimension of the film. It will be noted that even for a $100-\mathrm{mm}$ (4-in.) slit the elapsed time is three times the exposure time. With a between-the-lens shutter, for all speeds and apertures, the value of this ratio is 1 .

\begin{tabular}{|c|c|}
\hline Slit width & Ratio \\
\cline { 1 - 2 }$m m$ & \\
20 & 10 \\
40 & 5 \\
60 & 4 \\
80 & 3 \\
100 & 3 \\
\hline
\end{tabular}

\section{ACCESSORIES IN OPTICAL PATH OF CAMERA}

\section{FILTER}

(a) TYPES OF FILTERS

In airplane photography the exposures are commonly made through filters selected to absorb the light of shorter wave length in order to eliminate the loss of contrast produced by the blue haze that results from the scattering of light by the atmosphere. These filters are light yellow in color and, as the speeds of the available photographic emulsions increase, it is probable that filters of deeper color will be employed. (For special purposes deep-red filters and infrared sensitive film are now used.) The filters are of three materials (a) gelatine film, unsupported and unprotected, and usually placed within the lens, between the components, (b) gelatine film mounted with canada balsam between two plates of plane parallel glass, and (c) filters of colored glass, ground and polished with the surfaces plane and parallel.

The gelatine film is commonly mounted within the lens between the components in order to protect it from the effects of varying humidity. It is only the extreme thinness of the film that permits its use in this manner. Placing the filter between the components has the same effect, optically, as changing their spacing. Furthermore, the two surfaces of the filter, although approximately parallel, always depart greatly from planeness. Even under the most favorable conditions the use of a filter in this manner may be expected to affect the definition unfavorably. There is also the further danger that moisture will eventually reach the filter, even when mounted between the components, and that the filter will develop pronounced wrinkles that may ruin a series of exposures before their presence is detected. The necessary removal of the lens cells from the shutter for replacement of the gelatine filter from time to time is an additional undesirable feature. In view of these disadvantages, filters of gelatine film should not be used for photographic mapping purposes although, for experimental work in connection with the selection of a filter shade for a given emulsion, the use of gelatine filters is a convenient and inexpensive procedure.

The gelatine filter, mounted between glass plates, has one disadvantage. The balsam tends to deteriorate with age, the glass plates partially separate, and the filter assumes a nonuniform shade, or a feathery structure resulting from crystallization appears. 
A filter of colored glass performs exactly as the filter composed of a gelatine film cemented between two plates, but offers the advantage that it is entirely unaffected by humidity and does not deteriorate with age. For some purposes for which filters are required, it is not possible to obtain glass of the desired color and a cemented type of filter must be used. For eliminating atmospheric haze, satisfactory filters of colored glass are available, and this form of filter is preferable to the cemented type.

\section{(b) DISTORTION INTRODUCED BY FILTER}

Filters, either of colored glass or of the cemented type, are several millimeters thick and should never be placed between the components of a lens unless the manufacturer has designed the lens to be used in this manner. If the filter is placed between the lens and the focal plane, it will introduce appreciable amounts of negative distortion. The resulting distortion is of the same amount as that introduced by a pressure plate of the same thickness and this can be ascertained by reference to table 5, which gives the distortion at different angular distances from the axis for several glass thicknesses. Lenses have been built with a filter permanently mounted on the lens barrel, following the last component with the entire system designed to form a unit free from distortion. If a filter of good quality is mounted in front of a lens it will not introduce any distortion. If the surfaces of the filter are plane but not parallel it will act as a weak prism and deviate the rays slightly before they enter the lens. The angular deviation will be approximately equal to half the angle between the two surfaces. As a result of this deviation, the camera, when in a true vertical position, will make a photograph as though it were tilted through the angle of deviation produced by the prismatic filter. To illustrate this, the difference in thickness at opposite edges of a prism is $0.5 \mathrm{~mm}$ for a filter $50 \mathrm{~mm}$ square (a relatively large deviation from parallelism) a photograph taken with the camera in a vertical position will show a tilt of approximately 15 minutes. If the resulting photograph is properly adjusted in the plotting machine this will cause no error, as the effect of this tilt arising from the filter will be eliminated in the same manner that tilt arising from a nonvertical position of the camera is eliminated. If a filter is always to be used with a given camera, it is feasible to adjust the level with respect to the camera so that a picture without tilt is obtained when the level indicates horizontality.

(c) EFFECT OF FILTER UPON DEFINITION AND CAMERA CALIBRATION

It should be clearly understood that a filter, used with a photographic lens, is just as much a part of the optical system as any component of the lens and that defects in its quality are just as detrimental to good definition as are similar defects of the lens. Consequently, it is not good practice to purchase an expensive photographic objective with precisely polished surfaces and equip it with a filter of indifferent quality. An interference test, applied to the surfaces of the filter, should show plane surfaces of the same quality as lens surfaces, and the glass should be equally free from internal heterogeneities. If the filter has slightly spherical surfaces, its use will change the focal length and therefore affect the calibration of the camera but this $118273-39-8$ 
defect will not be appreciably present in filters of good quality. A wedge-shaped filter in front of the lens does not alter the position of the principal point. If a filter is of good quality and is to be used in front of the lens it is not necessary to calibrate the camera with the filter in position, although the National Bureau of Standards does calibrate cameras in this manner upon request. This method of test is advisable when it is suspected that the filter may impair the definition.

\section{PRESSURE PLATE}

The newer types of cameras are equipped with suction or pressure backs to hold the film plane during the exposure. Nevertheless, it seems advisable to discuss the action of the pressure plate of glass against which the film is pressed by a spring because many cameras with this equipment are still in use. There is a loss of light amounting to approximately 10 percent of that which would otherwise reach the photographic emulsion because of reflection at the two surfaces of the pressure plate. Unfortunately, a portion of this "lost" light will undergo multiple reflection at the two surfaces of the pressure plate and ultimately reach the emulsion as stray light, tending to reduce the contrast and detract from the brilliance of the image. If the pressure plate does not have surfaces of good quality, this will be somewhat detrimental to the quality of the image but the pressure plate is so close to the focal plane that its surfaces can affect definition relatively little as compared with the surfaces of the lens components.

Probably the most serious objection to the pressure plate has been the large amount of negative distortion that it introduces. This distortion is proportional to the thickness of the plate and increases from the center of the field outward. Values of the distortion for a pressure plate having the refractive index 1.52 are given in table $4 .{ }^{12}$

TABLE 4.-Linear distortion (in millimeters) produced by a plane parallel plate behind the lens

\begin{tabular}{|c|c|c|c|c|c|}
\hline \multirow{2}{*}{ Angular distance from center of field (degrees) } & \multicolumn{5}{|c|}{ Thickness of plate (in millimeters) } \\
\hline & 3 & 5 & 10 & 15 & 20 \\
\hline $\begin{array}{l}10 \\
30 \\
40\end{array}$ & $\begin{array}{r}0.00 \\
.00 \\
-.03 \\
-.10 \\
-.26\end{array}$ & $\begin{array}{r}0.00 \\
.00 \\
-.04 \\
-.16 \\
-.43\end{array}$ & $\begin{array}{r}0.00 \\
-.01 \\
-.08 \\
=.31 \\
-.85\end{array}$ & $\begin{array}{r}0.00 \\
-.02 \\
-.13 \\
-.47 \\
-1.28\end{array}$ & $\begin{array}{r}0.00 \\
-.02 \\
-.17 \\
-.63 \\
-1.71\end{array}$ \\
\hline
\end{tabular}

The large amount of distortion introduced by the pressure plate can be entirely and satisfactorily eliminated if the lens with which it is used has a corresponding amount of positive distortion. To design such a lens does not offer any great difficulty but this method of compensation for a pressure plate has not been employed. Such a procedure would necessitate the production of a greater variety of lenses for pressure plates of different thicknesses with consequent increased cost and the use of the suction or pressure back has become the more convenient and desirable construction.

Washington, October 24, 1938.

12 For a 7- by 9-inch negative the angular distances to the extreme corners of the field are $55.4^{\circ}, 44.0^{\circ}$, and $35.9^{\circ}$, respectively, for focal lengths of 100,150 , and $200 \mathrm{~mm}$. For a 9 - by 9 -inch negative the corresponding angular distances are $58.2^{\circ}, 47.1^{\circ}$, and $38.9^{\circ}$, respectively. 